\title{
Video Transmission in Block Vectors for Surveillance Applications
}

\author{
Papri Ghosh \\ Guest Lecturer in Sri Krishna College Under K.U
}

\begin{abstract}
Codecs perform encoding and decoding on a data stream or signal usually in the interest of compressing data. They scale, reorder, decompose and reconstitute perceptible images and sounds in information networks and electronic media. They are intimately associated with changes in the spectral density, the distribution of energy transported by sound and image in electronic media. Image registration is a crucial step in most image processing tasks for which the final result is achieved from a combination of various resources. In general most of the process followed by the image registration is a three step process. They are feature extraction, transform modeling, image merging. Obtaining salient, stable and distinguishable features increase the accuracy of the proposed registration process. Surveillance systems have been widely applied to many fields such as public safety, traffic monitoring and crime investigation. Recently intelligent surveillance technologies have shown their capability to improve on-line and off-line functions of surveillance systems. For off-line functions they can be applied to content based video retrieval. Motion detection plays an important role in intelligent surveillance systems. It is used to segment interested image areas and find possible moving objects in video data.
\end{abstract}

Keywords: feature extraction, crime investigation, block matching, histogram, camera tampering

\section{Introduction}

MPEG-2 designates a well established set of encoding and decoding procedures for digital audio and video formalized as a standard. The standard in fact defines a transport system rather than just a codec. Standards, as sociological work as argued , mix physical entities with conventional arrangements. Software implementations of such standards are known a s codecs. Video codecs for different standards(MPEG-1,MPEG-4, H.261,H.263, the important H.264, theora, dirac, DivX, MJPEG, WMV, RealVideo etc.) are strewn across the millions of sound and image associated with networked electronic media. Because codecs often borrow techniques and strategies of processing sound and image, their genealogy is tangled. Leaving aside the tangle of relations between different codecs and video technologies, even one codec, the well established and uncontentious MPEG-2 coding standard is extraordinarily complex. Algorithmically it combines several distinct compression techniques (converting signals from time domain to frequency domain using Discrete Cosine Transforms, quantization,Huffman and Run Length Encoding, block motion compensation), timing and multiplexing mechanisms, retrieval and sequencing techniques, many borrowed from the earlier, low bit-rate standard, MPEG-1. What appears on screen or what is heard increasingly depends on the techniques of lossy compression that MPEG-2 epitomizes. It generates artifacts (motion blocking, mosquito edging etc.) that affect at a deep level contemporary sensations of movement, color, light and time. Image registration is the process of overlaying images captured from the same scene but at different times and view points, or even by using different sensors. Therefore, it is a crucial step of most image processing tasks in which the final information is obtained from a combination of various data sources. These include image fusion, changes detection, robotic vision, archeology, medical imaging and multichannel image restoration. Typically, image registration is required in remote sensing applications such as change detection, multispectral classification, environmental monitoring. In this paper I have focused on the feature extraction step and have extracts the most dominant and stable features from images in a fully automatic manner. Due to the saliency of edge features in images and also their stability against environmental and illumination changes, here I have extracted image edges as primary features. Also I have extracted image edges as required control points. Consequently, more accurately extraction of edges leads to better control points detection which in turn improves the registration results. Motion detection plays an important role in intelligent surveillance systems. It is used to segment interested image areas and find possible moving objects in moving data. Global and local motion detectors are used to generate directional histograms from motion fields.

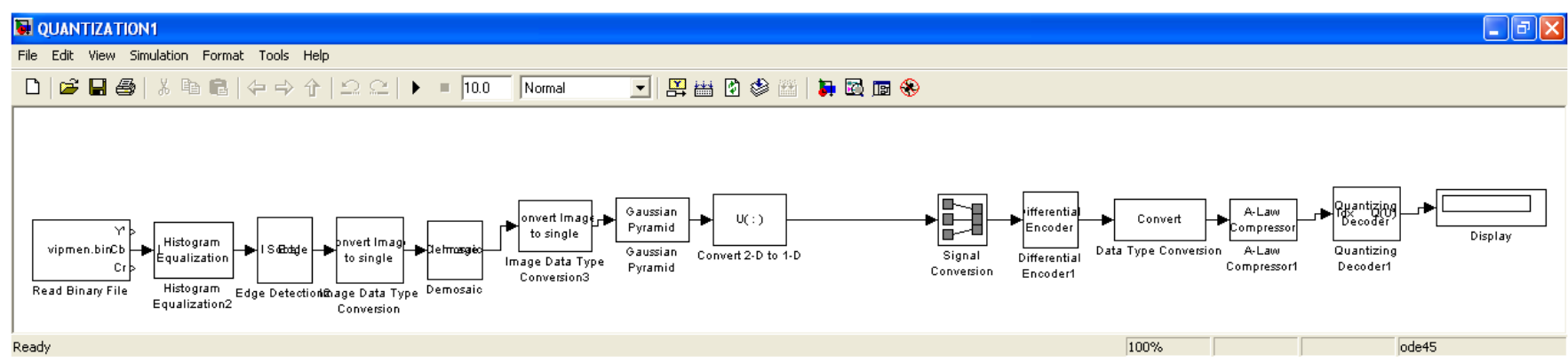

Figure 1: Image transmission into motion vectors

Volume 5 Issue 6, June 2016 www.ijsr.net 


\section{International Journal of Science and Research (IJSR) \\ ISSN (Online): 2319-7064 \\ Index Copernicus Value (2013): 6.14 | Impact Factor (2015): 6.391}

In an MPEG-2 video stream, images typically arrive at the codec as pixel-level luminance and chrominance values, and then go through several phases of encoding and decoding. These phases probe and restructure of the image quite deeply almost to the pixel level. In video and audio codecs, the DCT functions as a primary way of compressing or decompressing images or sound. Fourier transform or spectral analysis methods encompass a very wide range of computational problems in which data can be more easily analyzed and by analyzing signals that vary over time or space into a spectrum of frequencies that can be summed together to reconstitute the original signal. In the process of encoding a video sequence the MPEG-2 codec analyzes for each picture how blocks have moved and only transmits lists of motion vectors describing the movement of blocks in relation to a reference picture. The picture after encoding is nothing but a series of vectors describing what happens to blocks. Decoding the MPEG stream means turning these vectors back into arrangement of blocks moving between frames.

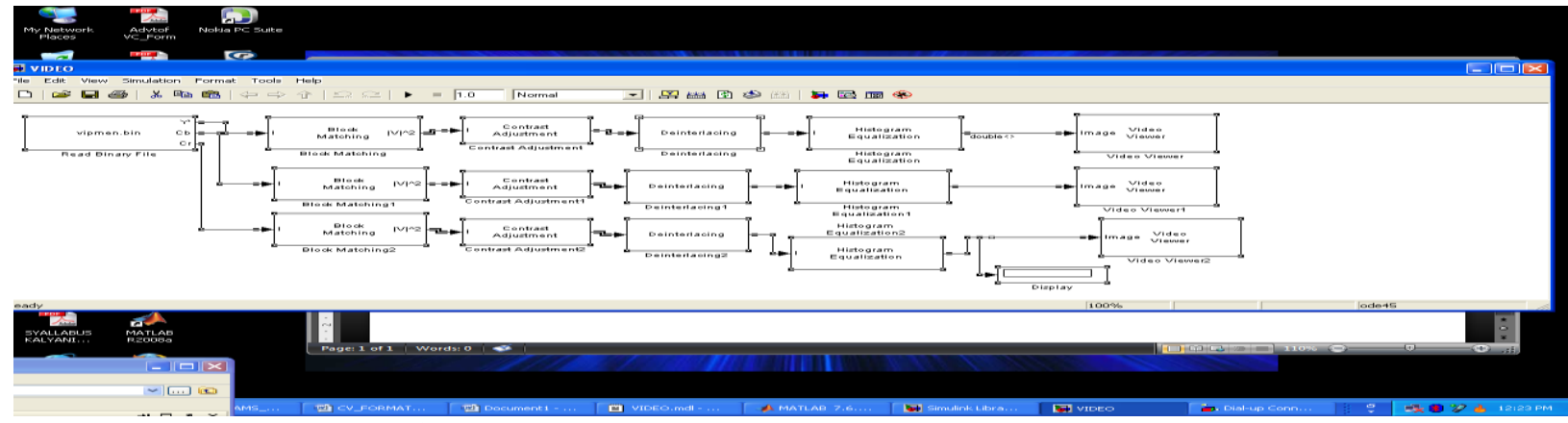

Figure 2: Block matching and Image recognisation system

\section{Feature Extraction}

To manually or preferably automatically extract salient and distinctive features such as closed boundary regions, edges and points. For further processes, these features can be represented by their point representatives who are called control points in the related literature.

There are many edge extraction methods reported in literature. They could be grouped in two main categories: point-wise and region-wise. In point-wise methods, only isolated pixels take part in the edge-extraction process. These include highpass and bandpass filtering as well as Robert, Sobel, Prewitt and Canny edge detectors. These methods have low computational costs but they cause ringing effects on extracted edges and also amplify high frequency noise. These methods result in disconnected edges too. Only the Canny edge detector of this group leads to connected extracted edges and this may lead to wrong edges and corners. In region-wise methods, the edges are extracted using a small neighbourhood of pixels. Rank based filters, statistical methods, Fourier-transform, Spline-interpolation, Laplacian-based and wavelet-based are samples of regionwise methods. All of these methods could extract salient and acceptable edges. However, they need heavy pre-processing leading to high computational costs. For example, in the rank based method, instead of raw pixel values, the value near median of a neighbourhood around the edge pixels is selected. In statistical methods, the distribution function of two neighbourhood objects is used to determine the edge pixels. Fourier transform based edge extraction methods use the frequency responses of edge pixels. In Splineinterpolation-based methods, first the edge pixels are interpolated along a Spline and then the edge pixels are extracted. In Laplacian and wavelet based methods, the edge extraction and its verification is done in a multi-resolution manner.
Gradient based corner detection techniques are more likely to respond to noise than their contour-based counterparts, and often perform quite poor results. In the proposed algorithm (Fig. 3) developed in Simulink 7.1 (MATLAB R2008a) the Harris corner detector uses the auto-correlation matrix to recognize the corners, but this method cannot be applied in multiscale mode. As such Harris corner detector is applied on a Gaussian pyramid. After point symmetry transform of edge pixels in the area of local window by the corner point as the center of symmetry, there is no intersection between the original edge pixels and the point symmetry transformed edge pixels for the corner. On the other hand, there must be at least one intersection point between them for the non-corner. After the extraction of corners by point symmetry transform of the edge image, distance weight function and phase weight function eliminate false corners for the corner verification and the exact localization of the real corners.

\subsection{Demosiacing}

Most digital cameras use a single sensor to record images and video. They use color filter arrays to capture one color band per pixel and interpolate colors to produce full RGB per pixel. This interpolation process is known as demosiacing

\subsection{Transforming}

A Fourier transform representation can be used to separate the various spatial scales of an image. Operating on this transform of an image we can no longer see local spatial features in a recognizable form. What is really needed is a representation that describes an image at multiple spatial resolutions and also preserves the local spatial structure that allows us to see the picture at each scale. Pyramid representations are ideal for this class of problems. 


\section{International Journal of Science and Research (IJSR) \\ ISSN (Online): 2319-7064}

Index Copernicus Value (2013): 6.14 | Impact Factor (2015): 6.391

The pyramid representation expresses an image as a sum of spatially band-passed images while retaining local spatial information in each band. A pyramid is created by low-pass filtering an image $\mathrm{G}_{0}$ with a compact two-dimensional filter. The filtered image is then sub- sampled by removing every other pixel and every other row to obtain a reduced image $G_{1}$. This process is repeated to form a Gaussian pyramid $G_{0}$, Gaussian pyramid $\mathrm{G}_{0}, \mathrm{G}_{1}, \mathrm{G}_{2}, \mathrm{G}_{3}, \ldots \mathrm{G}_{\mathrm{n}}$. $\mathrm{G}_{\mathrm{k}}(\mathrm{i}, \mathrm{j})=\Sigma \sum \mathrm{G}_{\mathrm{k}-\mathrm{l}}(2 \mathrm{i}+\mathrm{m}, 2+\mathrm{n}) \mathrm{k}=1, \mathrm{~N}$

Expanded image $\mathrm{G}_{\mathrm{k}, \boldsymbol{l}}$ is given by

$$
\left.\operatorname{Gkl}(\mathrm{i}, \mathrm{j}) \sum \sum \mathrm{G}_{\mathrm{k}, \mathrm{l}-1}[(2 \mathrm{i}+\mathrm{m} / 2,2 \mathrm{j}+\mathrm{n}) / 2)\right] \mathrm{f}(\mathrm{m}, \mathrm{n})
$$

Expanding G1 to the same size as G0 and subtracting yields the band-passed image. The original image can be reconstructed from the expanded band-pass images.

A fractal function includes both the basic form inherent in the object and its statistical or random properties. Fractals have the property of self-similarity over many different geometric scales. A fractal appears similar as the spatial scale is changed over many orders of magnitude. The pyramid breaks an image up into a sum of band-passed images plus a low-pass filtered image. If an inherently selfsimilar fractal image is decomposed into pyramid form, one would expect the band-passed images to look similar at each spatial frequency scale. Conversely, if similar patterns were entered into each spatial band of a pyramid, the reconstructed image should look like a fractal.

\subsection{Image Merging}

It is frequently desirable to combine several source images into a larger composite. Simple approaches to merging often create visible edge artifacts between regions taken from different source images. The blurred-edge effect is due to mismatch of low frequencies along the mosaic boundary, while the double-exposure effect is due to a mismatch in high frequencies.

\section{Experiment Results}

A colored video file was used for the simulation in Simulink. Sobel Edge operator was used to extract edges (Fig. 5). The image was demosiaced and then transformed using Gaussian Pyramid (Fig. 7). Image complement was used to reconstruct the image (Fig. 8). Although, the video image was obtained as fractals the image was tried to restore. The same operation was repeated for $\mathrm{Y}, \mathrm{Cb}$ and $\mathrm{Cr}$. Composite image (Fig. 6) (image blending) was tried for two $\mathrm{Y}$ and $\mathrm{Cr}$, and due to lack of place it was omitted for $\mathrm{Cb}$. Fig. 4 shows the histogram equalization of video block images.

There are some important technologies used in intelligent surveillance systems such as detection of fog and disturb visibility. Some research works have been done regarding detection of tampering with or modification of pre-recorded video that deal with data embedding and watermarking techniques. Histogram chromaticity difference is calculated to detect camera tampering. Camera tampering detection is based on comparison of recent and older frames of video data to determine the image dissimilarity. Using the algorithm developed as shown in Fig. 2 camera tampering of images could be detected. Fig. 1 shows the image transmission into motion vectors.

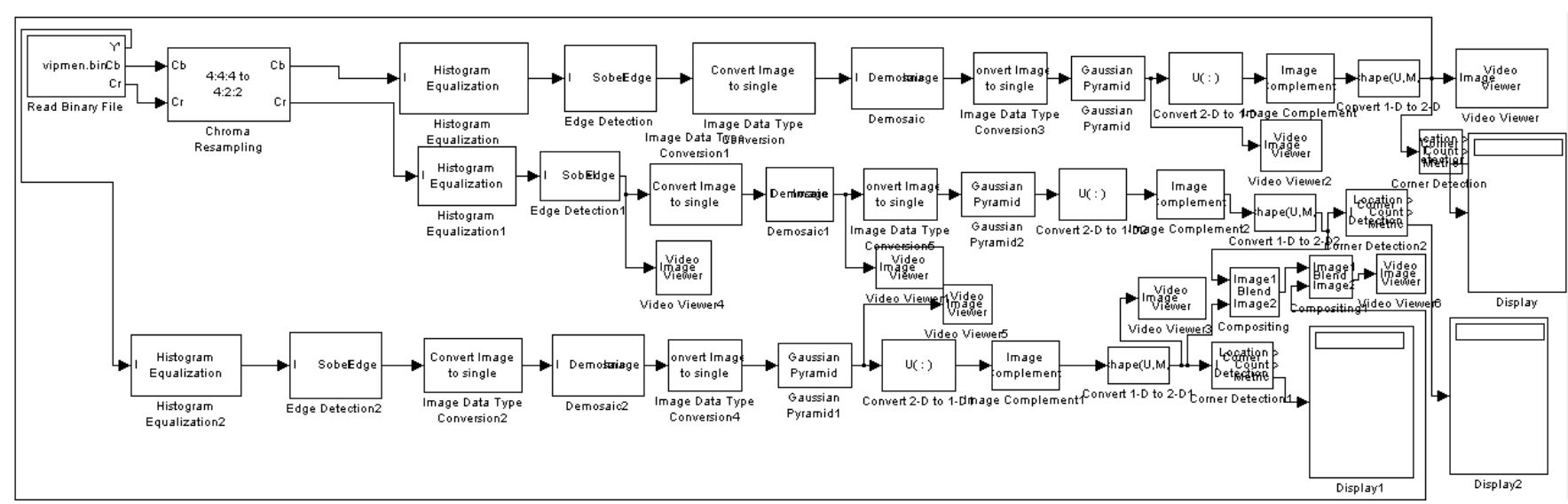

Figure 3: Video surveillance algorithm using MATLAB R2008a (Version 7.1)

Volume 5 Issue 6, June 2016 www.ijsr.net 


\section{International Journal of Science and Research (IJSR) \\ ISSN (Online): 2319-7064}

Index Copernicus Value (2013): 6.14 | Impact Factor (2015): 6.391

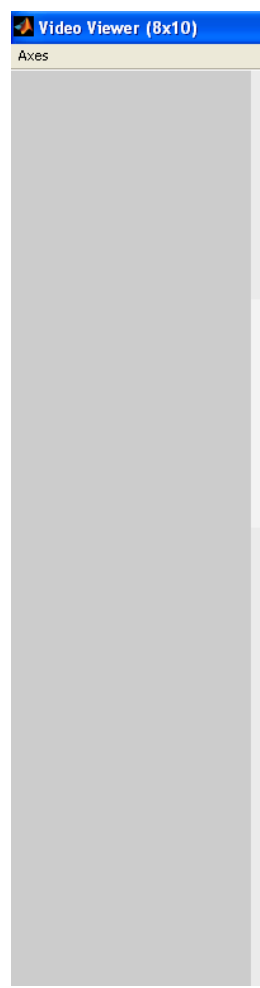

Figure 4: Histogram equalization for video images

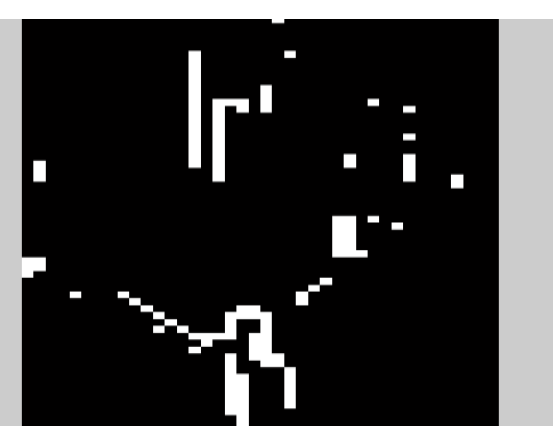

Figure 5: Sobel Edge Detection

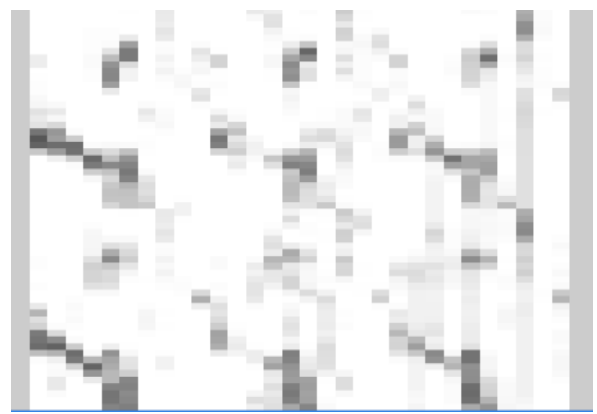

Figure 6: Composite reconstructed Image

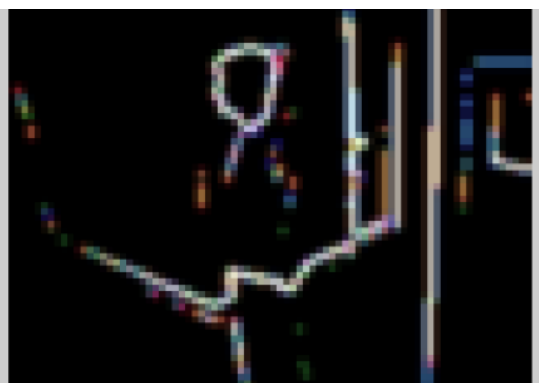

Figure 7: Image Transform using Gaussian Pyramid

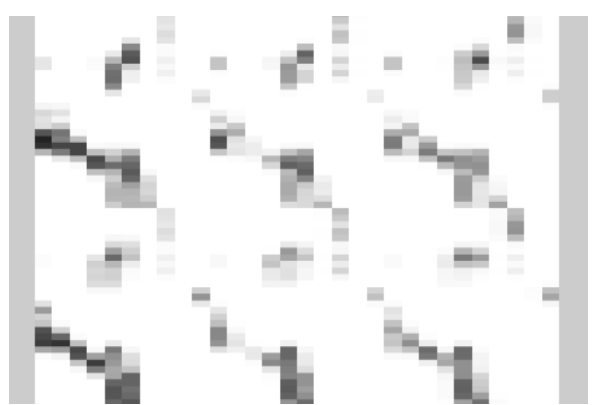

Figure 8: Reconstructed Image after Image Complement

\section{Conclusions}

In this paper a novel edge operator and a novel corner operator was introduced as an innovated algorithm. My proposed work could obtain the edges and the corners of gray-level images. Pyramidal representation seems particularly well-suited for making realistic looking computer graphic images on small systems.

\section{References}

[1] "Edge/Corner Programming" by H.S. Yazdi et al in International Journal of Signal Processing, Image Processing and Pattern recognition, on Vol.4, No.3, June 2011.

[2] Bayer, B.: Color Imaging array, In: U.S. Patent No. 3,971,065. (1976)

[3] Kovacs L, Utasi A, Szlavik Z, HAvasi L, Petras I, Sziranyi T Ditital Video Event Detector Framework for surveillance applications, Advanced video and signal based surveillance 2009, 565-570.

[4] Chiu-Chung Yu, Che-Yen Wen False alarm motion detection using the hierarchical block matching algorithm, Forensic Science Journal 2012, Vol 11, No.1.

Volume 5 Issue 6, June 2016 www.ijsr.net 